\title{
Dynamic stabilization versus fusion for treatment of degenerative spine conditions
}

\author{
Authors: Dean Chou ${ }^{1}$, Darryl Lau ${ }^{2}$, Andrea Skelly ${ }^{3}$, Erika Ecker $^{3}$ \\ Institutions: ${ }^{1}$ University of California, San Francisco, California, USA \\ ${ }^{2}$ University of Michigan Medical School, Ann Arbor, Michigan, USA \\ ${ }^{3}$ Spectrum Research Inc, Tacoma, Washington, USA
}

\section{ABSTRACT}

Study design: Comparative effectiveness review.

Study rationale: Spinal fusion is believed to accelerate the degeneration of the vertebral segment above or below the fusion site, a condition called adjacent segment disease (ASD). The premise of dynamic stabilization is that motion preservation allows for less loading on the discs and facet joints at the adjacent, non-fused segments. In theory, this should decrease the rate of ASD. However, clinical evidence of this theoretical decrease in ASD is still lacking. We performed a systematic review to evaluate the evidence in the literature comparing dynamic stabilization with fusion.

Clinical question: In patients 18 years or older with degenerative disease of the cervical or lumbar spine, does dynamic stabilization lead to better outcomes and fewer complications, including ASD, than fusion in the short-term and the long-term?

Methods: A systematic search and review of the literature was undertaken to identify studies published through March 7, 2011. PubMed, Cochrane, and National Guideline Clearinghouse Databases as well as bibliographies of key articles were searched. Two individuals independently reviewed articles based on inclusion and exclusion criteria which were set a priori. Each article was evaluated using a predefined quality-rating scheme.

Results: No significant differences were identified between fusion and dynamic stabilization with regard to VAS, ODI, complications, and reoperations. There are no long-term data available to show whether dynamic stabilization decreases the rate of ASD.

Conclusions: There are no clinical data from comparative studies supporting the use of dynamic stabilization devices over standard fusion techniques. 


\section{STUDY RATIONALE AND CONTEXT}

Spinal fusion is believed to accelerate the degeneration of the vertebral segment above or below the fusion site, a condition called adjacent segment disease (ASD) [1]. Dynamic stabilization systems have increased in popularity in the past decade as an alternative to fusion for treatment of degenerative spine conditions. In theory, they allow for less loading on the adjacent discs and facet joints and preserve adjacent segmental motion. Theoretically, this should lead to a decrease in ASD. However, it is unclear that such devices lead to better outcomes compared with traditional fusion, and it is unclear if it truly leads to a decrease in ASD.

\section{CLINICAL QUESTION}

In patients 18 years or older, with degenerative disease of the cervical or lumbar spine, does dynamic stabilization lead to better outcomes and fewer complications, including ASD, than fusion in the short-term and long-term?

\section{METHODS}

Study design: Comparative effectiveness review.

Search: PubMed, EMBASE, Cochrane Database of Systematic Reviews, bibliographies of key articles.

Dates searched: up through March 7, 2011.

Inclusion criteria: Studies directly comparing dynamic stabilization devices with traditional fusion (CoE I-III) in patients 18 years or older with degenerative spine disease (including spinal stenosis, spondylosis, spondylolisthesis, and/or degenerative disc disease).

Exclusion criteria: Studies in patients younger than 18 years, those with tumors, trauma, primary infection or inflammatory cause, fracture, Paget disease, osteochondrosis, congenital malformation, or visceral diseases, or previous lumbar surgery; studies comparing single versus multilevel dynamic stabilization without a comparison arm for fusion; studies of interspinous spacers (as sole device), dynamic devices, case series, non-human in vivo, in vitro, and biomechanical studies.

Outcomes: Visual analogue scale (VAS) for low back and lower leg pain; Oswestry disability index (ODI); ASD, reoperation, and other complications.
Analysis: Means and information on variation (eg, standard deviation or range) for continuous variables were abstracted from the report as available or if necessary, estimated from author figures and change scores calculated. The mean percentage improvement in the outcome score from baseline at each given follow-up time was calculated by dividing the change score by the baseline score to get the total percentage improvement. Rates of ASD, reoperation, and complications were calculated. Pooling of data was not done due to concerns regarding heterogeneity of treatments and populations as well as study quality.

Additional methodological and technical details are provided in the electronic supplemental material at www.aospine.orglebsj.

\section{RESULTS}

Most published reports were case series, in vitro or biomechanical studies. From a total of 79 citations retrieved, 14 were selected for full-text review (Fig 1). Of these, five met the inclusion criteria; however, duplicate papers on the same population were found $[2,3]$ and the most updated study was included for analysis [2]. Thus, four relatively small comparative studies are critically summarized [2-5], three of which were prospective. Populations were predominantly female (Table 1). Different dynamic stabilization systems were used including: the Cosmic dynamic pedicle screw-rod system, Twinflex rod system, Interspinous Soft Stabilization (ISS) with tension band system, and dynamic (hinged) pedicle screws (Table 2). All studies are class of evidence III.

Further details on the class of evidence rating for these studies can be found in the supplemental material at www.aospine.org/ebsj.

\section{VAS for low back pain and leg pain (Figs 2 and 3; Table 3)}

- The mean percentage improvement in VAS pain scores was generally similar for both treatment groups for both low back pain and leg pain across studies.

- No statistically significant differences between treatment groups was seen in mean VAS scores for either low back pain or leg pain at follow-up times reported by authors. All authors reported improvement in VAS scores relative to preoperative values for both treatment groups.

\section{Oswestry disability index (ODI) (Fig 4; Table 4)}

- Treatment groups were similar with regard to mean percentage improvement in ODI scores.

- No statistically significant differences between treatment groups for mean ODI were reported at any 
follow-up time. Both treatment groups experienced improvement in postoperative ODI scores compared with preoperative scores.

\section{Adjacent segment disease (Table 5)}

- In the three studies that reported ASD [2, 4, 5], ASD was not found in any of the patients treated with dynamic stabilization. In the fusion groups, occurrence of ASD ranged from $0 \%-9 \%$.

Fig 1 Results of literature search.

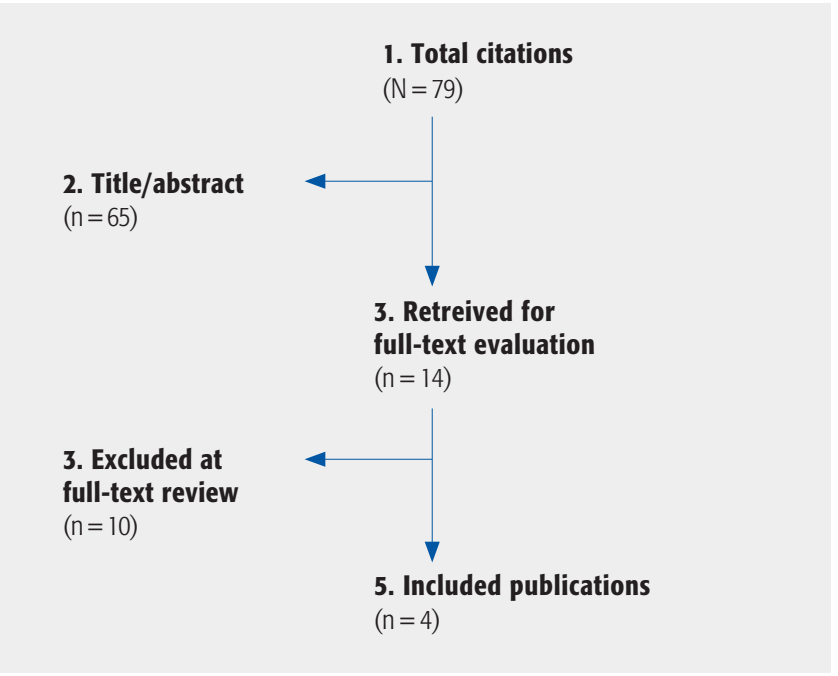

\section{Reoperation (Table 5)}

- Definitions of and indications for reoperation varied across studies, the extent to which these were device related was not always clear.

- In the dynamic groups, reoperation was reported in $0 \%-8 \%$ of patients primarily due to continued low back pain.

- In the fusion groups, reoperation was reported in $0 \%-9 \%$ of patients for various indications including ASD, deep infection, hematoma, and pseudarthrosis.

\section{Other complications (Table 5)}

- Reporting of complications varied across studies, and whether patients may have experienced more than one complication is generally not well delineated.

- In three studies, a higher number of complications overall was reported in the groups receiving fusion $[2,5,6]$. Complications included urinary tract infection, hematoma of the lumbar spine, deep infection, screw/rod breakage or loosening, radiolucency around screws, donor site pain, and fracture of the spinous process, pseudarthrosis, and malunion.

\section{CLINICAL GUIDELINES}

None found.

Fig 2 Percentage improvement from baseline in visual analogue scale scores for low back pain at 1, 2 and 4 years of follow-up in patients who underwent dynamic versus rigid fixation.

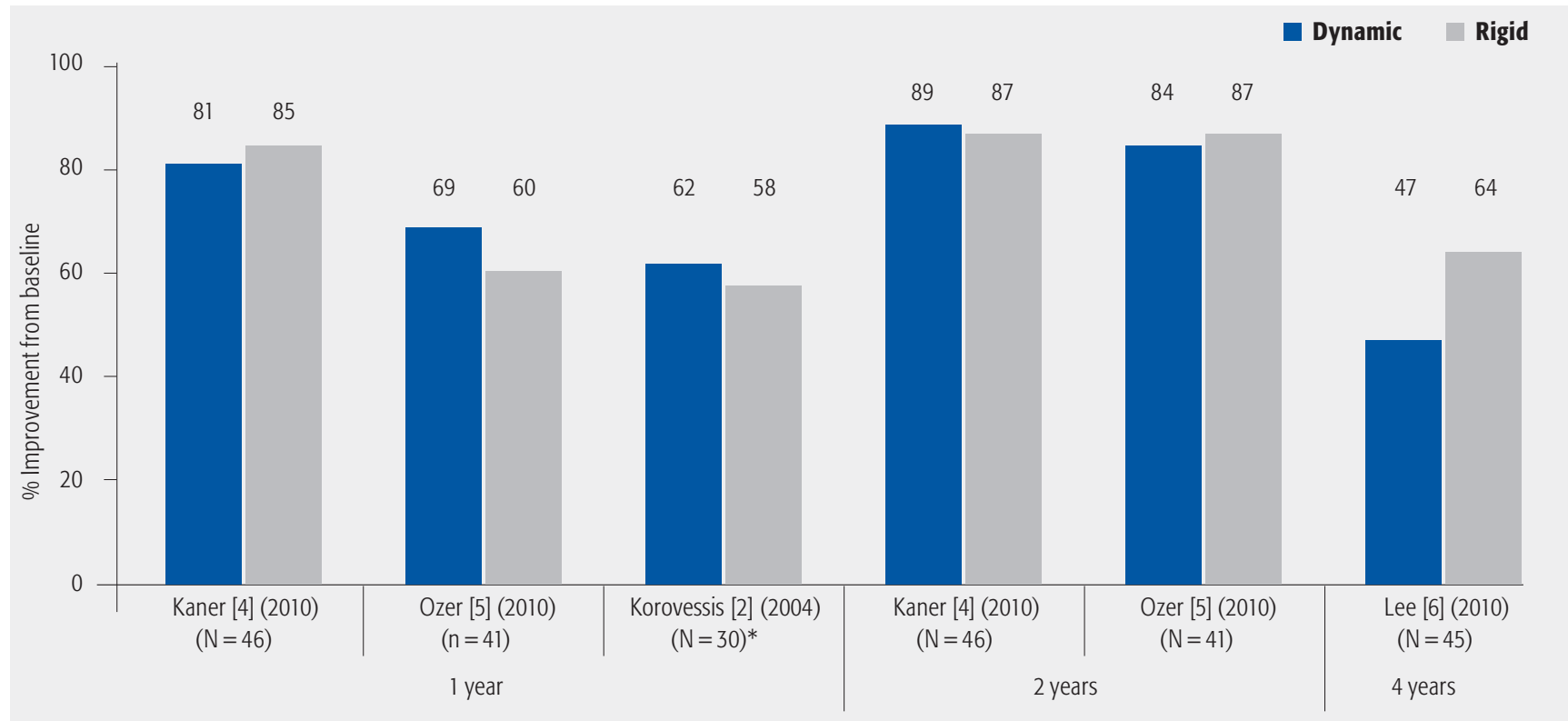

${ }^{*} \mathrm{~N}$ and outcome reflect only those patients in the rigid and dynamic fixation groups. The 15 patients who underwent dynamic fixation are not included in the analysis. 
Fig 3 Percentage improvement from baseline in visual analogue scale scores for leg pain at 1-year and 4-year follow-up in patients who underwent dynamic versus rigid fixation.

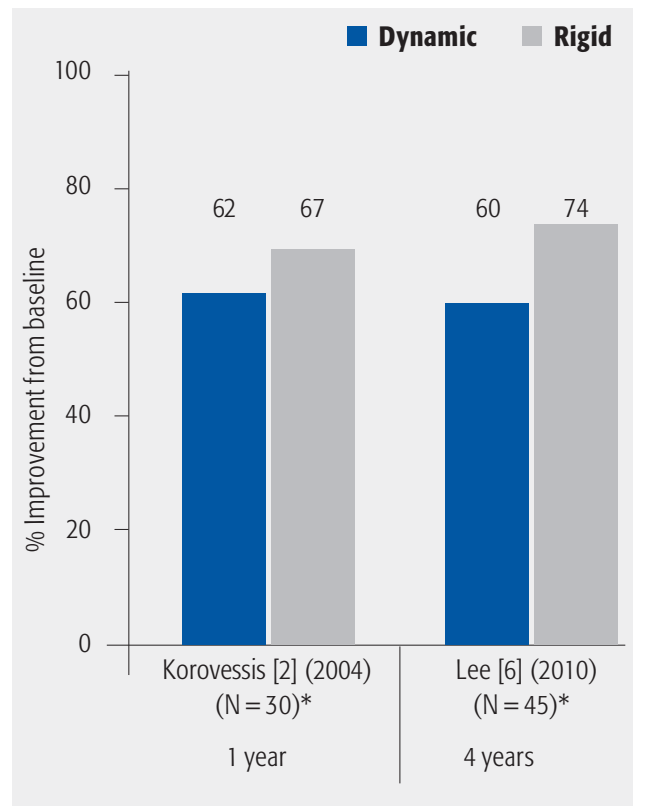

* $\mathrm{N}$ and outcome reflect only those patients in the rigid and dynamic fixation groups. The 15 patients who underwent semirigid fixation are not included in the analysis.
Fig 4 Percentage improvement from baseline in Oswestry disability index (ODI) scores at 1-, 2- and 4-year follow-up in patients who underwent dynamic versus rigid fixation.

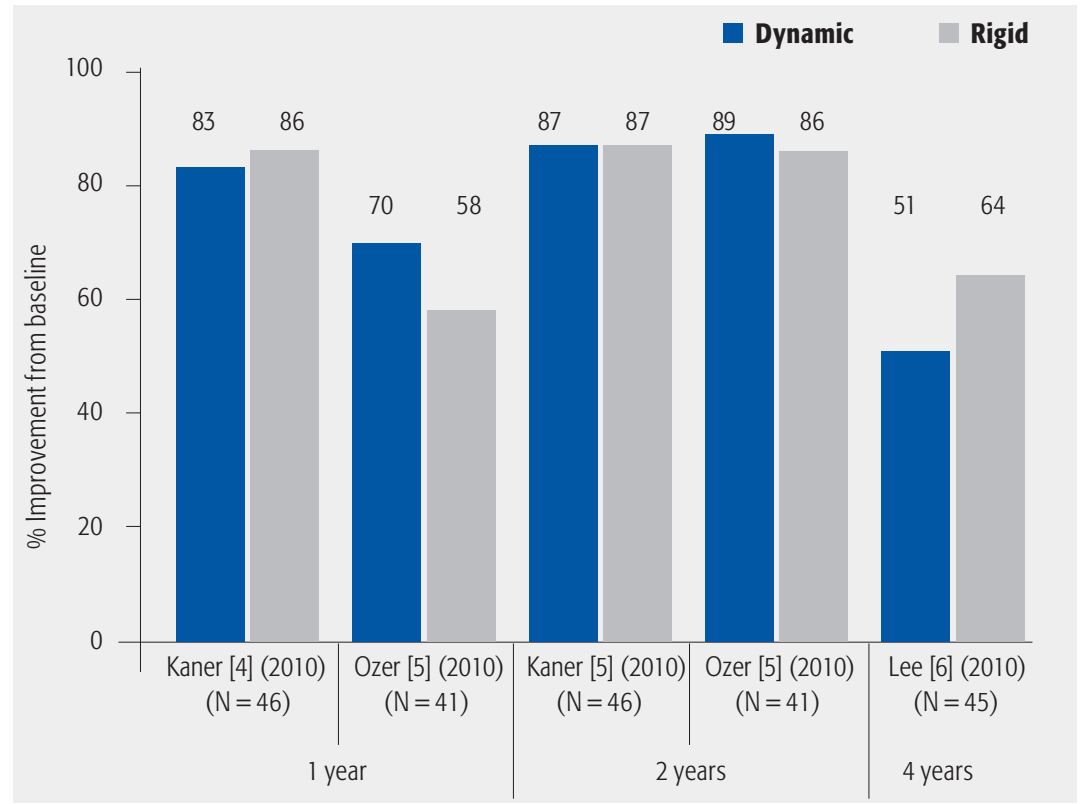

Table 1 Summary of population characteristics, intervention details, and inclusion/exclusion criteria for included studies comparing dynamic stabilization devices with fusion.*

\begin{tabular}{|c|c|c|c|c|c|c|}
\hline Author & $\begin{array}{l}\text { Study } \\
\text { design } \\
(\mathrm{CoE})\end{array}$ & $\begin{array}{l}\text { Follow-up (\% } \\
\text { followed up) }\end{array}$ & Demographics & Patient characteristics & Interventions & Inclusion/exclusion \\
\hline $\begin{array}{l}\text { Kaner et al } \\
{[4]} \\
(2010)\end{array}$ & $\begin{array}{l}\text { Prospective } \\
\text { cohort (III) }\end{array}$ & $\begin{array}{l}\text { Minimum } 24 \\
\text { mo (\% NR) } \\
\text { Dynamic: } \\
\text { mean } 38 \text { mo } \\
\text { (range, 24-55 } \\
\text { mo) } \\
\text { Fusion: mean } \\
44 \text { mo (range, } \\
26-64 \text { mo) }\end{array}$ & $\begin{array}{l}\text { Total } \\
\mathrm{N}=46 \\
\text { Male: } 28 \% \\
\text { Mean age }( \pm \mathrm{SD}) \text { : } \\
61.7 \pm 10.8 \text { y }(45-89) \\
\text { Dynamic } \\
\mathrm{n}=26 \\
\text { Male: } 23 \% \\
\text { Mean age }( \pm \mathrm{SD}) \text { : } \\
63.7 \pm 11.3 \text { y } \\
\text { Fusion } \\
\mathrm{n}=20 \\
\text { Male: } 35 \% \\
\text { Mean age }( \pm \mathrm{SD}) \text { : } \\
58.1 \pm 8.5 \text { y }\end{array}$ & $\begin{array}{l}\text { - Single-level grade I or II } \\
\text { degenerative } \\
\text { spondylolisthesis } \\
\text { causing central and/or } \\
\text { lateral recess syndrome } \\
\text { - Symptomatic, to include } \\
\text { leg pain and/or LBP or } \\
\text { hip pain due to } \\
\text { neurogenic claudication } \\
\text { - Failed medical } \\
\text { treatment before } \\
\text { enrollment }\end{array}$ & $\begin{array}{l}\text { - Surgical method was based on } \\
\text { patient preference } \\
\text { - All decompressions and fixations } \\
\text { conducted with microscopy and a } \\
\text { standard dorsal midline approach } \\
\text { using intraoperative image } \\
\text { intensifier } \\
\text { - Cosmic dynamic transpedicular } \\
\text { screws and rigid screws used in } \\
\text { the dynamic group } \\
\text { - Posterior and posterolateral } \\
\text { fusion together with PLIF were } \\
\text { performed by autograft in the } \\
\text { all-fusion group }\end{array}$ & $\begin{array}{l}\text { Included } \\
\text { - NR } \\
\text { Excluded } \\
\text { - Isthmic spondylolisthesis } \\
\text { - Degenerative spondylolisthesis at } \\
>1 \text { level } \\
\text { - History of previous lumbar fusion } \\
\text { surgery } \\
\text { - Spinal infections } \\
\text { - Systemic disease }\end{array}$ \\
\hline
\end{tabular}


Table 1 (cont) Summary of population characteristics, intervention details, and inclusion/exclusion criteria for included studies comparing dynamic stabilization devices with fusion.*

\begin{tabular}{|c|c|c|c|c|c|c|}
\hline Author & $\begin{array}{l}\text { Study } \\
\text { design } \\
\text { (COE) }\end{array}$ & $\begin{array}{l}\text { Follow-up (\% } \\
\text { followed up) }\end{array}$ & Demographics & Patient characteristics & Interventions & Inclusion/exclusion \\
\hline $\begin{array}{l}\text { Korovessis } \\
\text { et al [2] } \\
(2004)\end{array}$ & $\begin{array}{l}\text { Prospective } \\
\text { cohort (III) }\end{array}$ & $\begin{array}{l}\text { Mean } 47 \pm 14 \\
\text { mo (range, } \\
27-68 \mathrm{mo}) \\
(100 \%) \\
\text { (Random } \\
\text { selection of } \\
\text { patients) }\end{array}$ & $\begin{array}{l}\text { Dynamic } \\
n=15 \\
\text { Male: NR } \\
\text { Mean age }( \pm S D) \text { : } \\
62 \pm 10 \text { y } \\
\text { Rigid } \\
n=15 \\
\text { Male: NR } \\
\text { Mean age ( } \pm S D) \text { : } \\
65 \pm 9 \text { y } \\
\text { Semirigid } \\
N=15 \\
\text { Male: NR } \\
\text { Mean age ( } \pm S D) \text { : } \\
59 \pm 16 \text { y }\end{array}$ & $\begin{array}{l}\text { - Degenerative lumbar } \\
\text { spinal stenosis and } \\
\text { intractable neurogenic } \\
\text { claudication associated } \\
\text { with LBP } \\
\text { - Diagnoses included: } \\
\text { - Degenerative } \\
\text { spondylolisthesis } \\
\text { - Isthmic } \\
\text { spondylolisthesis } \\
\text { - Lumbar spondylosis } \\
\text { - Degenerative scoliosis }\end{array}$ & $\begin{array}{l}\text { - Rigid Segemental Contouring } \\
\text { System (SCS) with titanium alloy } \\
\text { rods and pedicle screws } \\
\text { - Semirigid Claris instrumentation } \\
\text { made from titanium alloy } \\
\text { comprised "semirigid" rods } \\
\text { connected to pedicle screws } \\
\text { - Twinflex device consisting of two } \\
\text { pairs of "dynamic" flexible } \\
\text { stainless steel rods and flat } \\
\text { connectors for each } \\
\text { instrumented level }\end{array}$ & $\begin{array}{l}\text { Included } \\
\text { - Symptomatic degenerative } \\
\text { lumbar spinal stenosis for } \geq 1 \text { y } \\
\text { - Acceptance of study protocol } \\
\text { Excluded } \\
\text { - Prior spine surgery } \\
\text { - Active infection } \\
\text { - Congenital deformity }\end{array}$ \\
\hline $\begin{array}{l}\text { Lee et al [6] } \\
(2010)\end{array}$ & $\begin{array}{l}\text { Retrospec- } \\
\text { tive cohort } \\
\text { (III) }\end{array}$ & $\begin{array}{l}\text { Overall: mean } \\
76.8 \text { mo }(\% \\
\text { NR) } \\
\text { Dynamic: } \\
\text { mean } \\
75.8 \pm 7.7 \text { mo } \\
\text { Rigid: } \\
\text { mean } \\
78.0 \pm 8.8 \text { mo }\end{array}$ & $\begin{array}{l}\text { Dynamic } \\
n=23 \\
\text { Male: } 26 \% \\
\text { Mean age: } 58.9 \pm 8.1 \\
y \\
\text { Rigid } \\
n=22 \\
\text { Male: } 36 \% \\
\text { Mean age: } 56.7 \pm 9.4 \\
y\end{array}$ & $\begin{array}{l}\text { - Grade } 1 \text { degenerative } \\
\text { spondylolisthesis with } \\
\text { stenosis } \\
\text { - Pain duration } \\
\text { - Dynamic: } 20.9 \pm 17.1 \\
\text { mo } \\
\text { - Rigid: } 17.5 \pm 12.2 \text { mo } \\
\text { - Smoking } \\
\text { - Dynamic: 4\% }(n=1) \\
\text { - Rigid: } 5 \%(n=1) \\
\text { - Level L3-L4 } \\
\text { - Dynamic: } 26 \%(n=6) \\
\text { - Rigid: } 18 \%(n=4) \\
\text { - Level L4-L5 } \\
\text { - Dynamic: } 74 \%(n=6) \\
\text { - Rigid: } 82 \%(n=4)\end{array}$ & $\begin{array}{l}\text { Lumbar decompression followed } \\
\text { by: } \\
\text { - ISS with a tension band system } \\
\text { - PLIF }\end{array}$ & $\begin{array}{l}\text { Included } \\
\text { - Chronic LBP and persistent leg } \\
\text { symptoms, including intermittent } \\
\text { neurogenic claudication } \\
\text { - } \geq 6 \text { mo conservative treatment } \\
\text { - Surgical indication symptomatic } \\
\text { stenosis with grade } 1 \\
\text { degenerative spondylolisthesis } \\
\text { defined as the upper vertebra } \\
\text { slipped forward }<25 \% \\
\text { - Single-level stabilization between } \\
\text { L3-L5 } \\
\text { Excluded } \\
\text { - Advanced segmental instability } \\
\text { such as ROM }>20^{\circ} \\
\text { - Prior surgery at any lumbar level } \\
\text { - Surgery at }>2 \text { levels } \\
\text { - Vertebral fracture } \\
\text { - Retrolisthesis } \\
\text { - Degenerative scoliosis }\end{array}$ \\
\hline $\begin{array}{l}\text { Ozer et al } \\
\text { [5] } \\
(2010)\end{array}$ & $\begin{array}{l}\text { Prospective } \\
\text { cohort (III) }\end{array}$ & $\begin{array}{l}24 \mathrm{mo} \\
\text { (\% NR) }\end{array}$ & $\begin{array}{l}\text { Dynamic } \\
\mathrm{n}=19 \\
\text { Male: } 26 \% \\
\text { Mean age: } 57.4 \\
\text { (17-80) y } \\
\text { Rigid } \\
\mathrm{n}=22 \\
\text { Male: } 45 \% \\
\text { Mean age: } 54.5 \\
\text { (20-86) y }\end{array}$ & $\begin{array}{l}\text { - Current smokers } \\
\text { - Dynamic, } n=9(47 \%) \\
\text { - Rigid, } n=6(27 \%) \\
\text { All patients were } \\
\text { stabilized at } 1 \text { level }\end{array}$ & $\begin{array}{l}\text { - Posterior lumbar pedicular } \\
\text { dynamic stabilization system; } \\
\text { facet joints and ligaments were } \\
\text { preserved from iatrogenic } \\
\text { damage during exposure } \\
\text { - Rigid posterior stabilization; facet } \\
\text { joints were decorticated to } \\
\text { promote fusion } \\
\text { - Hemilaminectomy, laminectomy, } \\
\text { and/or discectomy were } \\
\text { performed before rigid or } \\
\text { dynamic pedicle screw insertion, } \\
\text { done under image intensifier } \\
\text { - Surgical method chosen based } \\
\text { on patient preference } \\
\text { - All operations were performed } \\
\text { under general anesthesia in } \\
\text { knee-chest position using a } \\
\text { median line incision }\end{array}$ & $\begin{array}{l}\text { Included } \\
\text { - NR } \\
\text { Excluded } \\
\text { - Disc degeneration with } \\
\text { degenerative spondylolisthesis } \\
\text { - Failed nucleoplasty } \\
\text { - Recurrent disc herniation }\end{array}$ \\
\hline
\end{tabular}

${ }^{*}$ CoE indicates class of evidence; NR, not reported; SD, standard deviation; LBP, low back pain; PLIF, posterior lumbar interbody fusion; ISS, interspinous soft stabilization; and ROM, range of motion. 
Table 2 Overview of dynamic devices used in the included studies comparing dynamic stabilization with fusion.

\begin{tabular}{|c|c|c|c|}
\hline Author & Dynamic device & Description & Proposed design benefit(s) \\
\hline $\begin{array}{l}\text { Kaner et al [4] } \\
(2010)\end{array}$ & $\begin{array}{l}\text { Cosmic dynamic pedicular screw-rod } \\
\text { system (Ulrich GmbH \& co KG, Ulm, } \\
\text { Germany) }\end{array}$ & Hinged-pedicle screws combined with rigid rods & $\begin{array}{l}\text { Allows axial motion, reducing stress at the } \\
\text { bone-screw interface }\end{array}$ \\
\hline $\begin{array}{l}\text { Korovessis et al [2] } \\
(2004)\end{array}$ & $\begin{array}{l}\text { Twinflex rod system (Eurosurgical, } \\
\text { Ireland) }\end{array}$ & $\begin{array}{l}\text { Two pairs of flexible stainless steel rods ( } 2.5 \mathrm{~mm}) \\
\text { and flat connectors for each instrumented level } \\
\text { Longitudinal, smooth "semirigid" rods }(6 \mathrm{~mm}) \\
\text { connected transversely with a thin }(2 \mathrm{~mm}) \text { flat } \\
\text { connector to the pedicle screw head }\end{array}$ & $\begin{array}{l}\text { Flexibility of the longitudinal connections allows } \\
\text { the instrumentation to adapt to any screw } \\
\text { placement in both alignment and direction } \\
\text { Dynamic loading of the bone graft occurs as a } \\
\text { result of the elasticity of the construct }\end{array}$ \\
\hline Lee et al [6] (2010) & $\begin{array}{l}\text { Interspinous soft stabilization (ISS) with } \\
\text { tension band system (Ligament Vertebral } \\
\text { de Renfort; Cousine Biotech, } \\
\text { Wervicq-sud, France) }\end{array}$ & $\begin{array}{l}\text { Composed of polyester, polyethylene } \\
\text { terephthalate, and a central thread of barium, } \\
\text { platinum radiopaque silicone }\end{array}$ & $\begin{array}{l}\text { Achieve regional lumbar lordosis by placing the } \\
\text { motion segment into extension }\end{array}$ \\
\hline $\begin{array}{l}\text { Ozer et al [5] } \\
(2010)\end{array}$ & $\begin{array}{l}\text { Dynamic (hinged) pedicle screw } \\
\text { (Spahinaz, Medikon AS, Turkey) }\end{array}$ & Hinged-pedicle screws combined rigid rods & $\begin{array}{l}\text { Allows axial motion, reducing stress at the } \\
\text { bone-screw interface }\end{array}$ \\
\hline
\end{tabular}

Table 3 Mean visual analogue scale (VAS) $(10 \mathrm{~mm}$ ) pain scores* and change scores from baseline to follow-up in studies comparing dynamic stabilization devices with fusion.

\begin{tabular}{|c|c|c|c|c|c|c|c|c|c|c|}
\hline & \multicolumn{2}{|c|}{ Preoperative } & \multicolumn{2}{|l|}{$1 y$} & \multicolumn{2}{|l|}{$2 y$} & \multicolumn{2}{|l|}{$4 y$} & \multicolumn{2}{|c|}{ Change score } \\
\hline & Dynamic & Rigid & Dynamic & Rigid & Dynamic & Rigid & Dynamic & Rigid & Dynamic & Rigid \\
\hline \multicolumn{11}{|l|}{ VAS low back pain } \\
\hline \multirow[t]{2}{*}{ Kaner et al [4] (2010) } & $7.4 \pm 1.1$ & $7.9 \pm 1.1$ & $1.4 \pm 0.8$ & $1.2 \pm 0.7$ & $0.8 \pm 0.7$ & $1.0 \pm 1.0$ & & & $-6.0 \dagger$ & $-6.7 \dagger$ \\
\hline & & & & & & & & & $-6.6 \ddagger$ & $-6.9 \ddagger$ \\
\hline Korovessis et al [2] (2004) $)^{\S}$ & $5 \pm 1.4$ & $4 \pm 1.6$ & $1.9 \pm 1.2$ & $1.7 \pm 0.6$ & - & - & & & -3.1 & -2.3 \\
\hline Lee et al [6] (2010) & $6.4 \pm 1.9$ & $6.7 \pm 2.0$ & - & - & - & - & $3.4 \pm 2.6$ & $2.4 \pm 2.0$ & -3.0 & -4.3 \\
\hline \multirow[t]{2}{*}{ Ozer et al [5] (2010) } & 6.7 & 7.5 & 2.1 & 3 & 1.1 & 1.0 & & & $-4.6 \dagger$ & $-4.5 \dagger$ \\
\hline & & & & & & & & & $-5.6 \ddagger$ & $-6.5 \ddagger$ \\
\hline \multicolumn{11}{|l|}{ VAS lower leg } \\
\hline Korovessis et al [2] (2004) & $6.9 \pm 1.5$ & $7.6 \pm 1.9$ & $2.6 \pm 1.2$ & $2.5 \pm 1.5$ & - & - & & & -4.3 & -5.1 \\
\hline Lee et al [6] (2010) & $5.8 \pm 2.8$ & $7.3 \pm 1.9$ & - & - & - & - & $2.3 \pm 2.5$ & $1.9 \pm 2.2$ & -3.5 & -5.4 \\
\hline
\end{tabular}

* Higher scores indicate greater pain.

+ Change score from preoperative to 1 year.

₹ Change score from preoperative to 2 years.

$\S$ The 15 patients who received semirigid fixation are not included in the results.

Table 4 Mean Oswestry disability index scores* and change scores from baseline to follow-up in studies comparing dynamic stabilization devices with fusion.

\begin{tabular}{|c|c|c|c|c|c|c|c|c|c|c|}
\hline & \multicolumn{2}{|c|}{ Preoperative } & \multicolumn{2}{|l|}{$1 y$} & \multicolumn{2}{|l|}{$2 y$} & \multicolumn{2}{|l|}{$4 y$} & \multicolumn{2}{|c|}{ Change score } \\
\hline & Dynamic & Rigid & Dynamic & Rigid & Dynamic & Rigid & Dynamic & Rigid & Dynamic & Rigid \\
\hline \multirow[t]{2}{*}{ Kaner et al [4] (2010) } & $73.5 \pm 12.5$ & $75.7 \pm 9.5$ & $12.5 \pm 5.8$ & $10.3 \pm 4.6$ & $9.2 \pm 5.4$ & $10.2 \pm 7.8$ & - & - & $-61.0 \dagger$ & $-65.4 \dagger$ \\
\hline & & & & & & & & & $-64.3 \ddagger$ & $-65.5 \ddagger$ \\
\hline Lee et al [6] (2010) & $54.2 \pm 16.1$ & $59.7 \pm 17.9$ & - & - & - & - & $26.5 \pm 20.6$ & $21.7 \pm 14.7$ & -27.7 & -38 \\
\hline \multirow[t]{2}{*}{ Ozer et al [5] (2010) } & 64.5 & 62.0 & 19.5 & 26.2 & 7.4 & 8.6 & - & - & $-45.0 \dagger$ & $-35.8 \dagger$ \\
\hline & & & & & & & & & $-57.1 \ddagger$ & $-53.4 \ddagger$ \\
\hline
\end{tabular}

* Higher scores indicate greater disability.

† Change score from preoperative to 1 year.

$\S$ Change score from preoperative to 2 years. 
Table 5 Adjacent segment disease, reoperation, and other complications following dynamic versus rigid fixation.

\begin{tabular}{|c|c|c|}
\hline Author & $\begin{array}{l}\text { Dynamic } \\
\text { n (\%) }\end{array}$ & $\begin{array}{l}\text { Fusion } \\
\text { n (\%) }\end{array}$ \\
\hline $\begin{array}{l}\text { Kaner et al [4] } \\
(2010) \\
N=46\end{array}$ & $\begin{array}{l}\text { ASD*: } 0 \\
\text { Reoperation: } 2 \text { (8) } \\
\text { - Revision surgery for screw malposition within } 1 \text { month of index operation } \\
\text { - Fusion due to continued pain in year } 2 \\
\text { Other: NR }\end{array}$ & $\begin{array}{l}\text { ASD: } 1 \text { (5) } \\
\text { Reoperation: } 1 \text { (5) } \dagger \\
\text { Other: NR }\end{array}$ \\
\hline $\begin{array}{l}\text { Korovessis et al [2] } \\
(2004) \\
N=30 \S\end{array}$ & $\begin{array}{l}\text { ASD: } 0 \\
\text { Reoperation: } 0 \\
\text { Other } \\
\text { - UTI: } 1 \text { (7) } \\
\text { - Hematoma lumbar spine: } 1 \text { (7) } \\
\text { - Deep infection: } 1 \text { (7) } \\
\text { - Screw breakage: } 1 \text { (7)\| } \\
\text { - Rod breakage: } 2 \text { (14) } \\
\text { - Radiolucency around L5-S1 screws: } 2 \text { (14) } \\
\text { - Pseudarthrosis: } 0 \\
\text { - Malunion: } 0\end{array}$ & $\begin{array}{l}\text { ASD: } 0 \\
\text { Reoperation: } 2 \text { (14) } \ddagger \\
\text { Other } \\
\text { - Pneumonia: } 1 \text { (7) } \\
\text { - UTI: } 1 \text { (7) } \\
\text { - Hematoma lumbar spine: } 3 \text { (20) } \\
\text { - Hematoma donor site iliac crest: } 2 \text { (14) } \\
\text { - Deep infection: } 2 \text { (14) } \\
\text { - Donor site pain: } 4 \text { (27) } \\
\text { - Radiolucency around L5-S1 screws: } 3 \text { (20) } \\
\text { - Pseudarthrosis: } 0 \\
\text { - Malunion: } 0\end{array}$ \\
\hline $\begin{array}{l}\text { Lee et al [6] } \\
(2010) \\
N=45\end{array}$ & $\begin{array}{l}\text { ASD: NR } \\
\text { Reoperation: } 1 \text { (4) } \\
\text { - Dominant LBP with difficulty walking and standing resulting in ALIF } \\
\text { Other } \\
\text { - Deep wound infection: } 0 \\
\text { - Fracture of the spinous process: } 0\end{array}$ & $\begin{array}{l}\text { ASD: NR } \\
\text { Reoperation: } 0 \\
\text { Other } \\
\text { - Screw loosening: } 2 \text { (9) } \\
\text { - Screw breakage: } 1 \text { (5) }\end{array}$ \\
\hline $\begin{array}{l}\text { Ozer et al [5] } \\
(2010) \\
N=41\end{array}$ & $\begin{array}{l}\text { ASD: } 0 \\
\text { Reoperation: } 0 \\
\text { Other } \\
\text { - Screw loosening: } 2 \text { (11) } \\
\text { - Infection: } 0 \\
\text { - Chronic inflammation: } 0 \\
\text { - Fibrosis: } 0\end{array}$ & $\begin{array}{l}\text { ASD: } 2 \text { (9) } \\
\text { Reoperation: } 4(18)^{* *} \\
\text { - Pseudarthrosis } \\
\text { Other } \\
\text { - Broken screws: } 2 \text { (9) }\end{array}$ \\
\hline
\end{tabular}

* ASD indicates adjacent segment disease; NR, not reported; UTI, urinary tract infection; LBP, low back pain; and ALIF, anterior lumbar interbody fusion.

+ Performed in the patient with ASD.

₹ Performed for one of the patients with a deep infection and one evacuation for a hematoma in the lumbar spine.

$\S$ The 15 patients who received semirigid fixation are not included in results.

II Twelve months postoperatively.

" Eighteen months postoperatively.

\# Duration of pain 6-12 months postoperatively.

${ }^{* *}$ Two reoperations were in the two patients with ASD. 


\section{EVIDENCE SUMMARY}

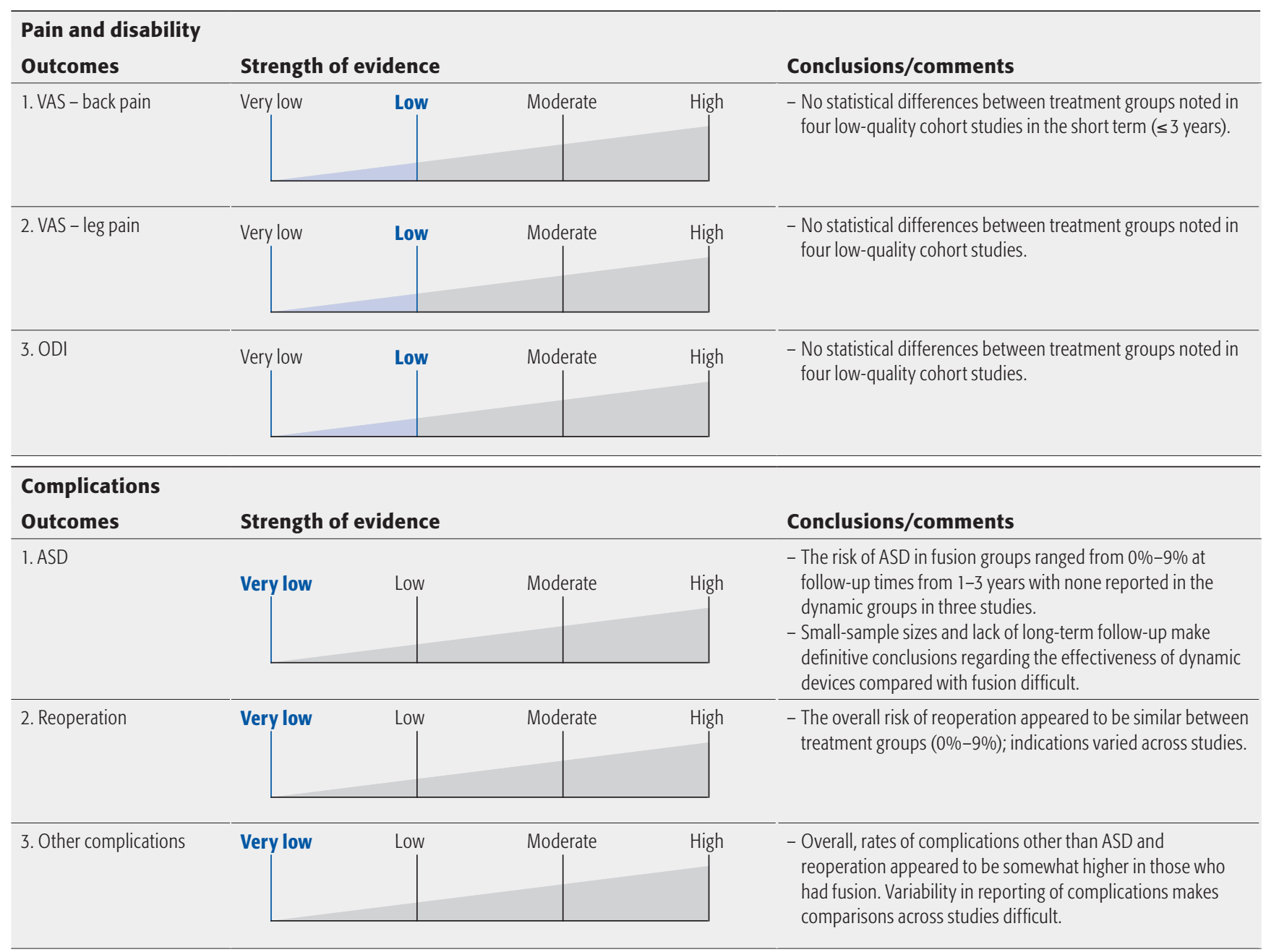




\section{DISCUSSION}

Conclusions from this review are limited by the following:

- There is heterogeneity with respect to dynamic stabilization devices used across studies. The mechanism by which these devices work and their designs vary significantly. Each device has a dynamic and a fixed component, and these are not the same in each device. For instance, one type of dynamic stabilization involves rigid rods, yet screws which flex and extend with reference to the tulip head. Another type is a posterior band which wraps around the spinous processes. Others involve normal pedicle screws with a non-rigid rod. This tremendous variation in types of dynamic stabilization makes it even more difficult for a direct comparison between dynamic stabilization and fusion. Thus, when interpreting the findings of this systematic review, such heterogeneity must be considered.

- Although from these findings it appears that there is no ASD in the dynamic stabilization group, the followup was simply not long enough. The longest follow-up study was 3 years, and given the small numbers there is simply not enough power to identify ASD in such a short follow-up. Thus, these factors should be carefully considered when interpreting the lower rate of ASD in the dynamic stabilization group.

- Inadequate description of numbers of eligible patients screened and loss to follow-up raise the possibility of significant selection bias in three of these studies, calling their validity into question. All studies were CoE III.
- No definition of clinically meaningful improvement in VAS or ODI was provided and the proportion of patients with such improvement not reported.

- Evidence on the influence of dynamic systems on rates of ASD is limited. Criteria for determining ASD were not well described in studies, length of follow-up in some studies may have been too short to detect ASD, and studies may have had insufficient power to detect differences between treatments on this outcome. The ASD status of those lost to follow-up is unknown.

- Surveillance for and definitions of complications varied across studies.

- The longest follow-up was a mean of 3 years; thus, evidence-based conclusions regarding the long-term effectiveness and safety are not possible.

- In November 2009, the US Food and Drug Administration (FDA) reviewed clinical data comparing the Dynesys dynamic stabilization device and fusion for degenerative lumbar conditions. Based on these data, the FDA rejected the widespread use of Dynesys [7]. The FDA has also ordered post-market surveillance for class II dynamic stabilization systems and components which are intended for bone fusion [8]. Given the data currently published and the executive decision by the FDA, it remains unclear as to whether dynamic stabilization improves outcome or decreases ASD.

- Although dynamic stabilization would seem to decrease the rate of ASD, there simply is not enough long-term data to support that this is in fact happening.

- There are no data from comparative studies to support the use of dynamic stabilization devices over standard fusion techniques. 


\section{REFERENCES}

1. Hilibrand AS, Carlson GD, Palumbo MA, et al (1999) Radiculopathy and myelopathy at segments adjacent to the site of a previous anterior cervical arthrodesis. J Bone Joint Surg Am; 81(4):519-528.

2. Korovessis $\mathbf{P}$, Papazisis Z, Koureas $\mathbf{G}$, et al (2004) Rigid, semirigid versus dynamic instrumentation for degenerative lumbar spinal stenosis: a correlative radiological and clinical analysis of short-term results. Spine; 29(7):735-742.

3. Korovessis P, Papazisis Z, Lambiris E (2002) The role of rigid vs. dynamic instrumentation for stabilization of the degenerative lumbosacral spine. Stud Health Technol Inform; 91:457-461.

4. Kaner $T$, Dalbayrak S, Oktenoglu $T$, et al (2010) Comparison of posterior dynamic and posterior rigid transpedicular stabilization with fusion to treat degenerative spondylolisthesis. Orthopedics; 33(5):309.

5. Ozer AF, Crawford NR, Sasani M, et al (2010) Dynamic lumbar pedicle screw-rod stabilization: two-year follow-up and comparison with fusion. Open Orthop J; 4:137-141.

6. Lee SH, Lee JH, Hong SW, et al (2010) Spinopelvic alignment after interspinous soft stabilization with a tension band system in grade 1 degenerative lumbar spondylolisthesis. Spine; 35(15):E691-701.

7. FDA Orthopedic and Rehabilitation Devices Panel. FDA Executive Summary for Zimmer Spine's Dynesys Spinal System. Available at: www.fda.gov/downloads/advisorycommittees/committeesmeetingmaterials/ medicaldevices/medicaldevicesadvisorycommittee/orthopaedicandrehabilitationdevicespanel/ucm188734.pdf. Accessed 2009.

8. FDA News Release. FDA Orders Postmarket Surveillance Studies on Certain Spinal Systems - FDA to request premarket clinical data for new versions of these devices. Available at: www.fda.gov/NewsEvents/Newsroom/PressAnnouncements/ucm185312.htm. Accessed October 5, 2009.

\section{EDITORIAL PERSPECTIVE}

The controversy surrounding the role and actual function of so-called "dynamic" fixation devices was reflected in the reviewers' comments. The devices in question are different from one another and defy simple biomechanical categorization. Their ability to provide long-term stabilization of a damaged operated-on segment remains to be seen-toggle loosening, implant breakage, foreign body reactions all await further clarification.

The other question that remains unanswered: Are patients with dynamic stabilization devices still mobile in their target level, or did they in fact inadvertently fuse? As more of these devices push on the market, a comparative biomechanical analysis using variety of loading mechanisms, including load to failure and fatigue loading together with monitoring of adjacent segment disc mechanics would appear to be a helpful first step to allow clinicians to understand the effects of the devices that are being offered for implantation. Again, at this time the basic first step, indications remain unclear. Then, the same can be said for the phenomenon of 'adjacent disc disease.' Is it patient borne, surgeon created, or a mixture of both? It is this first step that we need to understand before we can move ahead and try to identify preventative variables. 